\section{The origin of human retinoblastoma}

\section{The cellular origins of most human cancers remain unknown, but an analysis of embryonic retinal cells identifies differentiating cones as the cell of origin for the childhood cancer retinoblastoma. SEE LETTER P.385}

\section{ROD BREMNER \& JULIEN SAGE}

A $\mathrm{n}$ enduring mystery in our effort to understand most human cancers is the identities of the cells from which they arise. Attempts to define these 'cells of origin' have often used markers that are expressed in advanced tumours as a reference point. However, because cancer cells have, by definition, undergone a transformation from a normal to a diseased state, this approach is fatally flawed. By analogy, passengers disembarking from an aeroplane wearing winter clothes might look as if they had boarded in a cold country, but they could equally be arriving in a wintry location having set off from somewhere warm. In this issue, Xu et al. ${ }^{1}$ (page 385) take problem, identifying the cell type that gives rise to retinoblastoma by studying normal cells in the human retina.

Retinoblastoma is a childhood cancer of the retina that often serves as a model system for cancer studies. Indeed, work on this cancer led to the seminal discovery of the $R B 1$ gene $^{2}$, which encodes the retinoblastoma tumour-suppressor protein RB. To investigate the cell of origin of retinoblastoma, $\mathrm{Xu}$ and colleagues manipulated human embryonic retinal cells, and found that precursor cells destined to become cone photoreceptors are unusually sensitive to the loss of $R B 1$. The fact that cone precursors are differentiating cells committed to forming light-sensing retinal cells indicates that the cells of origin of human cancers do not necessarily have to be stem- or progenitor-cell types, as is often posited ${ }^{3,4}$. The authors purified human cones and showed that $\mathrm{RB}$ depletion in these cells, but not in other retinal populations, causes retinoblastoma when the cells are transplanted into recipient mice - a finding that resolves decades of $\operatorname{debate}^{5}$ (Fig. 1).

These data are compelling, but live imaging of early tumours from patients' eyes shows that lesions occur in the 'inner nuclear layer' of the retina ${ }^{6}$. This is the middle of three strata that comprise the retina, but cones are located in the outermost layer. Retinoblastomas might grow from displaced cone precursors in the inner nuclear layer. Alternatively, it is conceivable that a lack of RB causes a cell an alternative approach to the cell-of-origin in the inner nuclear layer to change its fate to become a cone or cone-like cell, because differentiating retinal cells are plastic. The susceptibility of purified cones to division and transformation following the loss of RB suggests that this idea is unlikely, although one should bear in mind that the cells used in these experiments have been dislodged from their normal milieu. There is also precedence for fate change in other cell lineages after $\mathrm{RB}$ loss $^{7}$.

What do the current results mean for mouse models of cancer? Mice are better protected from retinoblastoma than are humans - other tumour-suppressor genes must be deleted in genetically engineered mice in addition to the $R b 1$ gene to cause the cancer to develop ${ }^{8,9}$. As in humans, RB loss causes abnormal division of differentiating mouse retinal cells, but whereas $\mathrm{Xu}$ and co-workers observed that only cones are significantly affected in the human retina, all neuronal cell types are perturbed in that of the mouse $e^{8,9}$. The cell of origin for mouse retinoblastoma is also a differentiating retinal neuron, although of the amacrine (interneuron) lineage rather than the cone lineage . $^{8}$.

Amacrine and cone cells are generated in the retina at around the same developmental stage, and may thus share aspects of their gene-expression circuitry, especially early in their development. Indeed, the gene-expression patterns in human and mouse retinoblastoma are similar ${ }^{10}$, and there are also parallels in the genetic mutations that they harbour, such as deletions in the CDKN2A tumour-suppressor gene ${ }^{11}$. Furthermore, amacrine cells are located in the inner nuclear layer of the retina, where retinoblastoma emerges in humans. Thus, although there are differences in retinoblastomas between the two species, the numerous similarities make mouse models a valuable tool for future research and therapeutic testing.

One central issue in retinoblastoma and many other familial cancers is the striking specificity of tumour development. Why do patients with mutations in $R B 1$ develop tumours specifically in the eye before the age of five, even though the gene is expressed everywhere? The answer may lie in this latest study, and in previous observations made by the same group ${ }^{12}$. It seems that the molecular circuitry that is present in cone precursor cells renders them uniquely sensitive to cancerous transformation when $\mathrm{RB}$ is lost.

For instance, $\mathrm{Xu}$ et al. found evidence to suggest that high levels of the ubiquitin ligase enzymes SKP2 and MDM2, and of the cancercausing protein $\mathrm{N}-\mathrm{Myc}$, are crucial for cone precursors to begin proliferating without undergoing programmed cell death. Mouse amacrine cells seem to have similar circuits that sensitize them to the loss of RB, including the ability to resist cell death driven by the transcription factor E2F - a normal result of E2F expression following loss of RB function in mouse retinal cell types ${ }^{13}$. One interesting exception is the $\mathrm{p} 107$ protein, a relative of $\mathrm{RB}$ that has a tumour-suppressor role in mice ${ }^{13}$ but which the current study indicates can promote the development of cancer in human cone precursors harbouring $R B 1$ mutations.

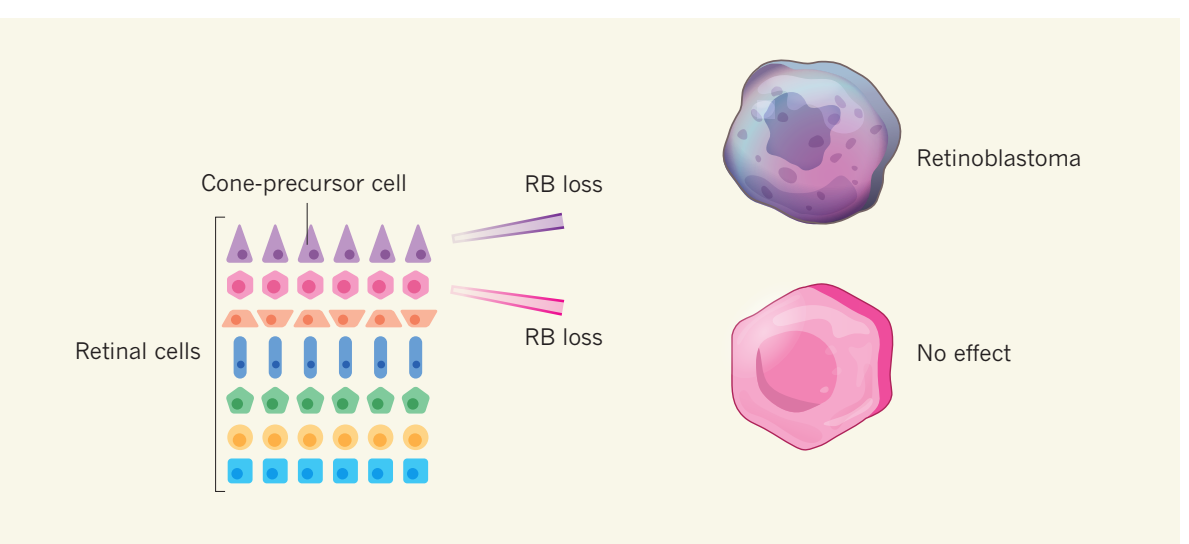

Figure 1 | The cone stands alone. Human retinal progenitor cells give rise to seven distinct cell types. Retinoblastoma develops specifically from differentiating cone precursors, owing to the molecular circuitry in these cells, which includes high expression of N-Myc, SKP2 and MDM2 proteins. This expression pattern permits the cells to proliferate and undergo a cancerous transformation when the tumour-suppressor protein RB is lost. In other retinal cell types, loss of RB either has no detectable effect or induces cell death (not shown). 
In conclusion, $\mathrm{Xu}$ and colleagues' fantastic work solves a controversial issue and provides a proof of principle for similar studies in other solid tumours. Once again, retinoblastoma acts as a model for the cancer field. Knowledge of the cell of origin for retinoblastoma (and other cancers) may help researchers to develop approaches for better diagnosis, earlier detection, and possibly chemoprevention. In addition, a better understanding of the molecular circuitry that renders cells susceptible to cancerous transformation may help to uncover Achilles heels in tumour cells. -

Rod Bremner is at Mount Sinai Hospital, Lunenfeld Tanenbaum Research Institute, Toronto, Ontario M5G 1X5, Canada. Julien Sage is in the Departments of Pediatrics and Genetics, Stanford University, Stanford, California 94305, USA. e-mails:bremner@lunenfeld.ca; julsage@stanford.edu

1. Xu, X. L. et al. Nature 514, 385-388 (2014)

2. Friend, S. H. et al. Nature 323, 643-646 (1986).

3. Visvader, J. E. Nature 469, 314-322 (2011).

4. Sage, J. Genes Dev. 26, 1409-1420 (2012).

5. Kyritsis, A. P., Tsokos, M., Triche, T. J. \& Chader, G. J. Nature 307, 471-473 (1984)

6. Rootman, D. B. et al. Br. J. Ophthalmol. 97, 59-65 (2013).

7. Calo, E. et al. Nature 466, 1110-1114 (2010).

8. Dyer, M. A. \& Bremner, R. Nature Rev. Cancer 5, 91-101 (2005).

9. Sangwan, M. et al. Oncogene 31, 5019-5028 (2012).

10.McEvoy, J. et al. Cancer Cell 20, 260-275 (2011).

11.Conkrite, K., Sundby, M., Mu, D., Mukai, S. \& Macpherson, D. J. Clin. Invest. 122, 1726-1733 (2012).

12.Xu, X. L. et al. Cell 137, 1018-1031 (2009).

13.Chen, D., Chen, Y., Forrest, D. \& Bremner, R. Cell Death Differ. 20, 931-940 (2013).

This article was published online on 24 September 2014.

\section{A historic experiment redesigned}

Large quasiparticles known as Rydberg excitons have been detected in a natural crystal of copper oxide. The result may find use in applications such as single-photon logic devices. SEE LETTER P.343

\section{SVEN HÖFLING \& ALEXEY KAVOKIN}

A $\mathrm{n}$ exciton is a quasiparticle in a solidstate system comprising an electron and a hole (the absence of an electron). It has an energy spectrum akin to that of a hydrogen atom, and so may be considered as an artificial hydrogen atom in a solid-state environment, with the hole playing the part of the hydrogen's proton. The concept of excitons was first formulated in the early 1930s by Yakov Frenkel ${ }^{1}$, who predicted their existence in molecular crystals. A few years later, Gregory Wannier ${ }^{2}$ and Nevill Mott $^{3}$ described these electron-hole bound states for inorganic semiconductors. In 1952, Evgeniy Gross and Nury Karryjew ${ }^{4}$ discovered these Wannier-Mott excitons experimentally in a copper oxide $\left(\mathrm{Cu}_{2} \mathrm{O}\right)$ semiconductor. Now, on page 343 of this issue, Kazimierczuk et al. ${ }^{5}$ report how they have redesigned this historic experiment to find excitons in a natural crystal of copper oxide. The excitons extend across some tens of billions of lattice sites of the crystal.

Gross and Karryjew's discovery marked the beginning of 'excitonics' - an area of solidstate physics that holds promise for applications in optoelectronics and in information and communication technologies ${ }^{6}$. For their studies, Gross and Karryjew selected crystals of copper oxide, and, using a spectrograph, identified eight dark lines in the material's transmission spectrum. Such absorption dips indicated the energies of optically induced transitions from the crystal's ground state to excited states with principal quantum numbers $n=2,3, \ldots 9$ (Fig. 1). The transition energies scaled with $n$ in a similar way to those of a hydrogen atom. This result proved that hydrogen-like quasiparticles, excitons, can be generated in these semiconductor crystals by photoabsorption.

In their study, Kazimierczuk et al. performed high-resolution transmission spectroscopy of an extremely high-quality natural crystal of copper oxide found at the Tsumeb mine in Namibia using laser light of tunable frequency and ultralow spectral linewidth (corresponding to roughly 1.2 megahertz). Taking advantage of the narrow linewidth of the laser and the high purity of the crystal, the authors have measured transmission spectra of the material with a spectral resolution of 5 nanoelectronvolts - an extremely high value for optical spectroscopy experiments. Analysis of the spectra revealed absorption lines associated with excitons with principal quantum numbers as large as $n=25$. The size of an exciton increases as $n^{2}$, with $n=25$ corresponding to a

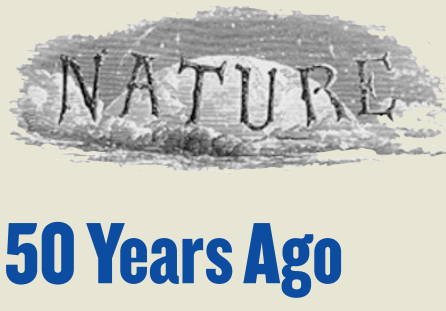

In general, the 'epidemic' process can be characterized as one of transition from one state (susceptible) to another (infective) where the transition is caused by exposure to some phenomenon (infectious material) ... People are susceptible to certain ideas and resistant to others. Once an individual is infected with an idea he may in turn, after some period of time, transmit it to others. Such a process can result in an intellectual 'epidemic' ... The development of the psychoanalytic movement in the early part of the twentieth century was in its way no less an 'epidemic' than was the outbreak of influenza in 1917 and 1918. One can argue similarly that Darwin and evolution, Cantor and set theory, Newton and mechanics, and so on, were examples of 'epidemics' in the world of scientific thought which were instigated by the introduction of a single infective into a population. From Nature 17 October 1964

100 Years Ago

At the present time astronomers have no available organisation by which the news of important astronomical discoveries can be quickly distributed to the leading observatories of the world, nor is there a bureau with which anyone making an important discovery can immediately communicate with the knowledge that the news will at once be circulated world wide. This condition of affairs is due to the fact that the recognised Central Bureau is at Kiel, in Germany, and that the state of war prevents the circulation of any such news ... There is little doubt that if the Royal Astronomical Society of Great Britain would undertake ... the task of receiving and disseminating astronomical information, this act would meet with the approval of astronomers all the world over. From Nature 15 October 1914 